\title{
Avaliação toxicológica pré-clínica do fitoterápico contendo Aristolochia cymbifera, Plantago major, Luehea grandiflora, Myrocarpus frondosus, Piptadenia colubrina (Cassaú Composto ${ }^{\circ}$ ) em ratos Wistar ${ }^{*}$
}

\author{
Pre-clinic toxicological evaluation of a phytotherapic containing Aristolochia cymbifera, Plantago major, \\ Luehea grandiflora, Myrocarpus frondosus, Piptadenia colubrina (Cassaú Composto ${ }^{\circ}$ ) in Wistar rats
}

\author{
Carina Rauber', Fernanda Bastos de Mello² \& João Roberto Braga de Mello²
}

\author{
RESUMO
}

O fitoterápico Cassaú Composto ${ }^{\circledR}$ é uma associação de extratos fluidos de Aristolochia cymbifera ("cassaú"), Plantago major L.("transagem"), Luehea grandiflora Mart.("açoita-cavalo"), Myrocarpus frondosus Allemão ("cabreúva"), Piptadenia colubrina Benth ("angico"). Avaliou-se a segurança deste fitoterápico através de estudos de toxicidade aguda e subcrônica, tendo como base a resolução $\mathrm{N}^{\circ} 90$, de 16 de março de 2004 da ANVISA. Para o teste de toxicidade aguda, ratos Wistar de ambos os sexos foram tratados por via oral com uma única dose de $26 \mathrm{ml} / \mathrm{kg}$, correspondendo a 20 vezes a dose terapêutica indicada pelo fabricante para seres humanos adultos. Os resultados revelaram haver sinais de toxicidade sistêmica com o aparecimento de ataxia, porém de forma transitória e reversível, não causando interferência no desenvolvimento ponderal dos animais, nos consumos de água e ração, nas produções de urina e fezes, bem como alterações macroscópicas nos órgãos dos animais. Avaliou-se também a exposição a doses repetidas do fitoterápico. Constituiram-se 4 grupos experimentais (10 animais/sexo/dose), onde administrou-se por via oral a ratos Wistar, durante 30 dias, doses diárias de $1,3 \mathrm{ml} / \mathrm{kg}$, $6,5 \mathrm{ml} / \mathrm{kg}$ e $13 \mathrm{ml} / \mathrm{kg}$, respectivamente a dose terapêutica indicada pelo fabricante para seres humanos adultos, 5 vezes, e 10 vezes a dose terapêutica, além de um grupo controle, onde administrou-se o veículo do fitoterápico. Os resultados revelaram ausência de toxicidade sistêmica, fundamentados na ausência de alterações hematológicas e bioquímicas sangüíneas, bem como peso e análises histopatológicas dos órgãos, nos diferentes grupos. Concluiu-se que a utilização do fitoterápico nas doses e períodos referidos pode ser considerado segura.

Descritores: Aristolochia cimbifera, Plantago major, Luehea grandiflora, Myrocarpus frondosus, Piptadenia colubrina.

\section{ABSTRACT}

The phytotherapic Cassaú Composto ${ }^{\circledR}$ is an association of fluid extracts of Aristolochia cymbifera, Plantago major L., Luehea grandiflora Mart., Myrocarpus frondosus Allemão and Piptadenia colubrina Benth (Cassaú Composto ${ }^{\circledR}$ ). The safety of the phytotherapic was evaluated through studies of acute and sub-chronic toxicity, being based in the resolution $\mathrm{N}^{\mathrm{o}} 90$, March $16^{\text {th }}, 2004$ from ANVISA. For the test of acute toxicity Wistar rats of both sexes were treated orally with a single dose of $26 \mathrm{ml} / \mathrm{kg}$, which corresponds to 20 times the therapeutic dose indicated by the producer for adult humans. The results revealed that there are signals of systemic toxicity including transitory and reversible ataxy, no interference in the development of weight gain in the animals, in water and feed consume, in the production of urine and feces, neither macroscopic alterations in the animals' organs. It was also evaluated the exposition of repeated doses of the phytotherapic (sub-chronic toxicity). Four experimental groups (10 animals/sex/dose) were oraly treated during 30 days with daily doses of $1,3 \mathrm{ml} / \mathrm{kg}$, $6,5 \mathrm{ml} / \mathrm{kg}$ and $13 \mathrm{ml} / \mathrm{kg}$, respectively the therapeutic dose indicated to humans, 5 times, and 10 times the therapeutic dose, and a control group, receiving the phytotherapic vehicle. The results revealed the absence of systemic toxicity, based in the absence of hematological and blood biochemical alterations, as well as weigh and histopatological analysis of organs, in the different groups. It was concluded that the utilization of the referred phytotherapic in the mentioned doses and periods might be considered secure.

Key words: Aristolochia cimbifera, Plantago major, Luehea grandiflora, Myrocarpus frondosus, Piptadenia colubrina. 


\section{INTRODUÇÃO}

A utilização de extratos vegetais com fins terapêuticos (fitoterápicos), tem sido crescente, tanto em países desenvolvidos, como em países em desenvolvimento, através de uma intensa ascensão da indústria farmacêutica [3]. De acordo com a Organização Mundial da Saúde (OMS), os medicamentos fitoterápicos são definidos como produtos com fins medicinais que contém derivado ativo obtido das plantas. Segundo a ANVISA (Agência Nacional de Vigilância Sanitária), os fitoterápicos ainda são caracterizados pelo conhecimento da eficácia e dos riscos de seu uso, assim como pela constância e reprodutibilidade de efeitos e controle de sua qualidade.

Plantas medicinais em contraste com medicamentos sintéticos, muitas vezes tem sido chamadas de não tóxicas, devido as suas origens naturais e por longos períodos de utilização na medicina tradicional [15]. Os estudos toxicológicos tem a finalidade de avaliar a idéia errônea de que produtos fitoterápicos, por serem naturais, são isentos de efeitos tóxicos ou adversos, e que o uso popular de plantas medicinais serve como validação da eficácia destes medicamentos [6,13].

O medicamento fitoterápico Cassaú Composto ${ }^{\circledR}$ é um "tônico reconstituinte" indicado como auxiliar no tratamento da inapetência e da debilidade física, não existindo relatos sobre sua atividade toxicológica, especialmente de toxicidade aguda e subcrônica.

O trabalho teve como objetivo avaliar a toxicidade pré-clínica oral aguda e de doses repetidas (subcrônica) deste fitoterápico em ratos, tendo como base o guia para a realização de estudos de toxicidade pré-clínica de fitoterápicos [1].

\section{MATERIAIS E MÉTODOS}

\section{Animais}

Foram utilizados ratos albinos Wistar de ambos os sexos, com idade inicial de 120 dias com massa corporal de $304,70 \pm 28,88 \mathrm{~g}$ (machos) e $218,04 \pm 15,16 \mathrm{~g}$ (fêmeas), provenientes do Centro de Criação e Experimentação de Animais de Laboratório da UFRGS (CREAL). Os animais foram mantidos no Biotério Setorial do Departamento de Farmacologia do Instituto de Ciências Básicas da Saúde (ICBS), com condições constantes de umidade, temperatura $\left(21^{\circ} \mathrm{C} \pm 2\right)$ e ciclo de luz claro/escuro de 12 horas (claro das 7 às 19h). Foram alimentados com ração comercial e água $a d l i$ bitum durante todo o período experimental. Os animais foram aclimatados às condições do Biotério Setorial por um período mínimo de 5 dias.

\section{Medicamento Fitoterápico}

O medicamento fitoterápico Elixir Cassaú Composto $^{\circledR 1}$ utilizado nos experimentos foi fornecido pelo laboratório Cibecol, sob lote $\mathrm{n}^{\circ}$ 040131(fabricado em junho de 2004 com validade de 3 anos) com registro no Ministério da Saúde sob número 1.0077.0004.

Composição quali-quantitativa do fitoterápico:

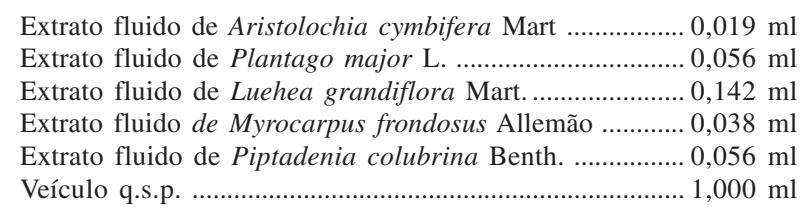

\section{Protocolo Experimental}

\section{- Experimento 1: Toxicidade oral aguda}

Foi administrada dose correspondente a 20 vezes a dose terapêutica preconizada pelo fabricante para seres humanos adultos com peso de $\pm 70 \mathrm{~kg}$, correspondendo a dosagem de 2,6 ml/100g. Dois grupos de machos e fêmeas ( $n=6 /$ sexo) foram formados onde um grupo recebeu em dose única por via oral através de sonda gástrica flexível, o volume correspondente do fitoterápico, e o outro grupo recebeu a mesma dose, porém do veículo do fitoterápico. Durante os 14 dias do período de experimentação, machos e fêmeas foram mantidos individualmente em gaiolas metabólicas $(25 \times 22 \times 21 \mathrm{~cm})$. Estas gaiolas metabólicas foram utilizadas com o intuito de se avaliar diariamente e individualmente cada animal quanto aos consumos de ração e água, e também produções de fezes e urina.

Os animais foram observados quanto a sinais de toxicidade (alteração da locomoção, insuficiência respiratória e cardíaca, piloereção, sialorréia, alteração do tônus muscular, hipnose, convulsões, hiperexcitabilidade do sistema nervoso central, contorções abdominais, tremores, diarréia, letargia e coma) durante as primeiras 24 horas, nos seguintes tempos após a administração: 15 e 30 minutos, 1 e 4 h. Após estes tempos os animais foram observados diariamente uma vez ao dia durante os 14 dias do experimento, onde os resultados foram anotados em ficha específica. Além disso, diariamente os animais foram avaliados quanto a consumo de ração e água (g), massa corporal (g), produção de urina e fezes $(\mathrm{g})$.

\section{- Experimento 2: Toxicidade de doses repetidas (subcrônico)}

Para este teste foram constituídos 4 grupos (10/sexo/dose) onde administrou-se por via oral a ratos Wistar, durante 30 dias, doses diárias de $1,3 \mathrm{ml} / \mathrm{kg}$, 
$6,5 \mathrm{ml} / \mathrm{kg}$ e $13 \mathrm{ml} / \mathrm{kg}$, respectivamente a dose terapêutica indicada pelo fabricante para seres humanos adultos, 5 vezes, e 10 vezes a dose terapêutica, além de um grupo controle, onde foi administrado o veículo do fitoterápico. Em todos os grupos tratados com o fitoterápico, assim como o grupo controle, foi avaliada a massa corporal diária $(\mathrm{g})$, consumo diário de ração $(\mathrm{g})$, consumo diário de água $(\mathrm{ml})$, análise macroscópica dos órgãos, peso relativo (\%) das vísceras no dia do sacrifício (fígado, rins, pulmão, coração, esôfago, estômago, intestinos, pâncreas, adrenais, tireóides e órgãos sexuais), assim como também foram avaliados o aparecimento de sinais de toxicidade sistêmica como perda de peso progressiva e diminuição na ingestão de água e ração. Exames complementares como análise histopatológica dos órgãos, hemograma completo e análise bioquímica sangüínea (sódio, potássio, gama-glutamiltranspeptidase, aminotransferases, fosfatase alcalina, uréia, creatinina, ácido úrico, colesterol total, triglicerídeos, glicose, proteínas totais e bulirrubina total) foram realizados nos grupos tratados com a maior dose (13 ml/kg/dia) e controle. No grupo tratado com a menor dose $(1,3 \mathrm{ml} / \mathrm{kg} / \mathrm{dia})$ e controle também foi avaliado produção de urina e fezes.

Os dados foram avaliados por análise de variância (ANOVA), análise de variância de medidas repetidas (ANOVA de MR) e teste de Bonferroni. Foi utilizado o programa SPSS para Windonws 11.0 e o EXCEL. As variáveis quantitativas como o desenvolvimento ponderal dos animais de cada grupo, consumo de água e de ração, produção de urina e fezes, foram comparadas através da análise de variância de medidas repetidas.

As variáveis quantitativas como análise bioquímica, massa relativa dos órgãos de machos e fêmeas foram comparadas através da análise de variância de uma via (ANOVA). Quando necessário efetuou-se o pós-teste de Bonferroni para identificação dos grupos estatisticamente diferentes $[4,14]$.

As diferenças foram consideradas significativas sempre que $\mathrm{p}<0,05$, com nível de confiança de $95 \%$.

\section{RESULTADOS}

\section{Toxicidade aguda}

Os resultados revelaram haver sinais de toxicidade sistêmica com o aparecimento de ataxia, porém de forma transitória e reversível, não causando interferência no desenvolvimento ponderal dos animais, nos consumos de água e ração, nas produções de urina e fezes, bem como alterações macroscópicas nos órgãos dos animais.

\section{Toxicidade de doses repetidas (subcrônica)}

Não foram observadas alterações significativas nos ratos tratados com qualquer das três dosagens do fitoterápico em comparação ao grupo controle. As oscilações diárias no consumo de água e ração e produção de fezes e urina, não mantiveram relação com o desenvolvimento ponderal dos animais. Não foram detectadas alterações morfológicas nos órgãos internos de machos e fêmeos, cuja massa relativa não diferiu significativamente do grupo controle, com qualquer das dosagens do fitoterápico (Tabelas 1 e 2).

O quadro hematológico dos animais tratados com o fitoterápico não diferiu significativamente quando comparados ao grupo controle. As séries leucocitárias de machos e fêmeas, apresentaram determinadas diferenças significativas quando comparadas ao grupo controle, porém dentro dos limites normais para a espécie (Tabelas 3 e 4).

A avaliação bioquímica das fêmeas, valores de ácido úrico, creatinina e potássio apresentaram-se aumentados, ao contrário das enzimas alanina-aminotransferase (ALT), aspartato-aminotranferase (AST) e fosfatase alcalina (FA), bem como gama-glutamiltranferase (GGT) e proteínas totais que apresentaramse diminuídas, quando comparado ao grupo controle (Tabela 4). Os machos apresentaram redução significativa na atividade da enzima fosfatase alcalina (FA), bem como creatinina e proteínas totais, contrariamente ao valor do ácido úrico, cujo valor aumentou (Tabelas 5 e 6$)$.

\section{DISCUSSÃO}

O índice terapêutico (IT) por definição representa a relação entre a dose letal para 50\% dos indivíduos $\left(\mathrm{DL}_{50}\right)$ e a dose eficaz para $50 \%$ dos indivíduos $\left(\mathrm{DE}_{50}\right)$. Para uma maior segurança farmacológica, uma definição mais conservadora é a que relaciona a dose letal para $1 \%$ dos indivíduos com a dose eficaz para 99\% dos indivíduos, ou ainda a dose capaz de provocar efeitos adversos (LOEL) em relação à maior dose incapaz de produzir efeitos adversos (NOEL). A relação de 10 vezes, representa uma relativa segurança para a substância química ou para o fitoterápico, ou baixa toxicidade $[11,12]$. Considerando que para o teste de toxicidade subcrônica, a utilização de doses diárias de $1,3 \mathrm{ml} / \mathrm{kg}, 6,5 \mathrm{~m} / \mathrm{kg}$ e $13 \mathrm{ml} / \mathrm{kg}$, respectivamente 
Tabela 1. Massa relativa dos órgãos das ratas tratadas durante 30 dias com fitoterápico Cassaú Composto ${ }^{\circledR}$ com três diferentes doses, CC1 1,3 ml/kg/dia, (CC5) 6,5 ml/kg/dia, (CC10) $13 \mathrm{ml} / \mathrm{kg} / \mathrm{dia}$, e um grupo controle CC (13 ml/kg/dia) tratado com o veículo do fitoterápico. São apresentados os valores percentuais médios \pm epm.

\begin{tabular}{llllc}
\hline Massa relativa dos órgãos $(\%)$ & $\mathbf{C C} 1(\mathbf{n}=10)$ & $\mathbf{C C 5}(\mathbf{n = 1 0})$ & $\mathbf{C C 1 0}(\mathbf{n}=10)$ & $\mathbf{C C}(\mathbf{n = 1 0})$ \\
\hline Adrenal direita & $0,023 \pm 0,004$ & $0,020 \pm 0,001$ & $0,013 \pm 0,001$ & $0,018 \pm 0,001$ \\
Adrenal esquerda & $0,023 \pm 0,003$ & $0,020 \pm 0,001$ & $0,016 \pm 0,001$ & $0,020 \pm 0,001$ \\
Baço & $0,272 \pm 0,015$ & $0,307 \pm 0,001$ & $0,291 \pm 0,002$ & $0,269 \pm 0,001$ \\
Coração & $0,362 \pm 0,013$ & $0,363 \pm 0,002$ & $0,331 \pm 0,003$ & $0,348 \pm 0,001$ \\
Esôfago & $0,035 \pm 0,005$ & $0,032 \pm 0,002$ & $0,030 \pm 0,003$ & $0,034 \pm 0,002$ \\
Estômago & $0,688 \pm 0,021$ & $0,661 \pm 0,024$ & $0,667 \pm 0,004$ & $0,701 \pm 0,004$ \\
Fígado & $3,678 \pm 0,044$ & $3,654 \pm 0,004$ & $3,507 \pm 0,004$ & $3,505 \pm 0,004$ \\
Ovário direito & $0,037 \pm 0,005$ & $0,043 \pm 0,007$ & $0,039 \pm 0,005$ & $0,037 \pm 0,007$ \\
Ovário esquerdo & $0,037 \pm 0,004$ & $0,047 \pm 0,010$ & $0,035 \pm 0,005$ & $0,037 \pm 0,009$ \\
Pulmão & $0,635 \pm 0,030$ & $0,619 \pm 0,021$ & $0,569 \pm 0,013$ & $0,547 \pm 0,018$ \\
Rim direito & $0,348 \pm 0,009$ & $0,353 \pm 0,053$ & $0,324 \pm 0,022$ & $0,338 \pm 0,028$ \\
Rim esquerdo & $0,354 \pm 0,010$ & $0,348 \pm 0,072$ & $0,322 \pm 0,032$ & $0,332 \pm 0,034$ \\
Tireóide direita & $0,006 \pm 0,001$ & $0,006 \pm 0,079$ & $0,007 \pm 0,058$ & $0,006 \pm 0,042$ \\
Tireóide esquerda & $0,006 \pm 0,001$ & $0,005 \pm 0,097$ & $0,006 \pm 0,060$ & $0,005 \pm 0,055$ \\
Útero & $0,425 \pm 0,017$ & $0,407 \pm 0,364$ & $0,335 \pm 0,257$ & $0,351 \pm 0,304$ \\
\hline n = número de animais por grupo. & & & &
\end{tabular}

Tabela 2. Massa relativa dos órgãos dos ratos tratados durante 30 dias com fitoterápico Cassaú Composto ${ }^{\circledR}$ com três diferentes doses, CC1 1,3 ml/kg/dia, (CC5) 6,5 ml/kg/dia, (CC10) $13 \mathrm{ml} / \mathrm{kg} / \mathrm{dia}$, e um grupo controle CC (13 ml/kg/dia) tratado com o veículo do fitoterápico. São apresentados os valores percentuais médios \pm epm.

\begin{tabular}{llllc}
\hline Massa relativa dos órgãos $(\%)$ & $\mathbf{C C} 1(\mathbf{n}=\mathbf{1 0})$ & $\mathbf{C C 5}(\mathbf{n}=\mathbf{1 0})$ & $\mathbf{C C 1 0}(\mathbf{n}=10)$ & $\mathbf{C C}(\mathbf{n}=10)$ \\
\hline Adrenal direita & $0,011 \pm 0,001$ & $0,011 \pm 0,001$ & $0,011 \pm 0,001$ & $0,009 \pm 0,001$ \\
Adrenal esquerda & $0,010 \pm 0,001$ & $0,011 \pm 0,001$ & $0,013 \pm 0,001$ & $0,009 \pm 0,001$ \\
Baço & $0,318 \pm 0,013$ & $0,335 \pm 0,023$ & $0,313 \pm 0,010$ & $0,286 \pm 0,010$ \\
Coração & $0,335 \pm 0,012$ & $0,321 \pm 0,006$ & $0,331 \pm 0,008$ & $0,314 \pm 0,007$ \\
Epidídimo direito & $0,294 \pm 0,012$ & $0,269 \pm 0,016$ & $0,247 \pm 0,012$ & $0,263 \pm 0,011$ \\
Epidídimo esquerdo & $0,294 \pm 0,009$ & $0,297 \pm 0,017$ & $0,256 \pm 0,015$ & $0,259 \pm 0,011$ \\
Esôfago & $0,025 \pm 0,001$ & $0,030 \pm 0,003$ & $0,032 \pm 0,002$ & $0,028 \pm 0,002$ \\
Estômago & $0,553 \pm 0,023$ & $0,539 \pm 0,016$ & $0,539 \pm 0,018$ & $0,570 \pm 0,017$ \\
Fígado & $3,543 \pm 0,077$ & $3,379 \pm 0,048$ & $3,346 \pm 0,052$ & $3,382 \pm 0,055$ \\
Próstata & $0,170 \pm 0,020$ & $0,129 \pm 0,010$ & $0,129 \pm 0,015$ & $0,118 \pm 0,007$ \\
Pulmão & $0,535 \pm 0,012$ & $0,505 \pm 0,007$ & $0,489 \pm 0,016$ & $0,468 \pm 0,007$ \\
Rim direito & $0,336 \pm 0,006$ & $0,328 \pm 0,011$ & $0,317 \pm 0,006$ & $0,310 \pm 0,005$ \\
Rim esquerdo & $0,339 \pm 0,006$ & $0,331 \pm 0,009$ & $0,307 \pm 0,006$ & $0,306 \pm 0,009$ \\
Testículo direito & $0,508 \pm 0,024$ & $0,523 \pm 0,012$ & $0,536 \pm 0,012$ & $0,501 \pm 0,010$ \\
Testículo esquerdo & $0,493 \pm 0,019$ & $0,540 \pm 0,016$ & $0,539 \pm 0,014$ & $0,507 \pm 0,010$ \\
Tireóide direita & $0,005 \pm 0,001$ & $0,004 \pm 0,001$ & $0,005 \pm 0,001$ & $0,004 \pm 0,001$ \\
Tireóide esquerda & $0,004 \pm 0,001$ & $0,004 \pm 0,001$ & $0,004 \pm 0,001$ & $0,003 \pm 0,001$ \\
Vesícula seminal & $0,238 \pm 0,021$ & $0,231 \pm 0,016$ & $0,190 \pm 0,009$ & $0,214 \pm 0,010$ \\
\hline
\end{tabular}

$\mathrm{n}=$ número de animais por grupo. 
Tabela 3. Valores médios do leucograma ( \pm erro padrão da média) em ratas tratadas com fitoterápico Cassaú Composto ${ }^{\circledR}$ na dose de $13 \mathrm{ml} / \mathrm{kg} / \mathrm{dia}$ (CC10) e um grupo controle tratado com o veículo do fitoterápico na dose de $13 \mathrm{ml} / \mathrm{kg} / \mathrm{dia}(\mathrm{CC})$.

\begin{tabular}{|c|c|c|}
\hline Variáveis & $\operatorname{CC} 10(n=5)$ & $C C(n=5)$ \\
\hline $\begin{array}{l}\text { Contagem global } \\
\text { de Leucócitos }\left(\mathrm{mm}^{3}\right)^{\mathrm{a}}\end{array}$ & $4280 \pm 374,4$ & $5780 \pm 940,3$ \\
\hline Neutrófilos bastonados (\%) & nihil & nihil \\
\hline Neutrófilos segmentados (\%) & $29 \pm 2,2$ & $33,8 \pm 1,0$ \\
\hline Eosinófilos (\%) & $0,6 \pm 0,2$ & $2,2 \pm 0,4$ \\
\hline Basófilos (\%) & nihil & nihil \\
\hline Linfócitos (\%) & $68,2 \pm 2,4^{*}$ & $59,8 \pm 2,1$ \\
\hline Monócitos (\%) & $2,2 \pm 0,7$ & $4,2 \pm 0,9$ \\
\hline \multicolumn{3}{|c|}{$\begin{array}{l}\mathrm{n}=\text { número de animais por grupo; }{ }^{\mathrm{a} A} \text { contagem global de leucócitos } \\
\text { representa valores absolutos. A contagem de neutrófilos, eosinófilos, } \\
\text { basófilos, linfócitos e monócitos representa valores relativos (percen- } \\
\text { tuais) à contagem global de leucócitos; "Diferença estatisticamente } \\
\text { significativa em relação ao grupo controle }(\mathrm{p}<0,05) \text {. }\end{array}$} \\
\hline
\end{tabular}

Tabela 5. Níveis séricos da atividade do ácido úrico, ALT, AST, bilirrubina, creatinina, proteínas totais, triglicerídeos, colesterol total, FA, glicose, potássio, sódio, uréia e GGT em ratas tratadas com o fitoterápico Cassaú Composto ${ }^{\circledR}$ na dose de $13 \mathrm{ml} / \mathrm{kg} / \mathrm{dia}$ (CC10) e um grupo controle tratado com o veículo do fitoterápico na dose de $13 \mathrm{ml} / \mathrm{kg} / \mathrm{dia}$ (CC). Dados expressos pela média absoluta (valores bioquímicos) \pm epm.

\begin{tabular}{lcc}
\hline Variáveis & CC10 $(\mathbf{n}=5)$ & CC $(\mathbf{n}=5)$ \\
\hline Ácido úrico $(\mathrm{mg} / \mathrm{dl})$ & $3,50 \pm 0,45^{*}$ & $1,56 \pm 0,21$ \\
ALT (U/l) & $40,64 \pm 4,47^{*}$ & $64,60 \pm 3,53$ \\
AST (U/l) & $83,59 \pm 12,08^{*}$ & $126,60 \pm 6,90$ \\
Bilirrubina total (mg/dl) & $0,59 \pm 0,02$ & $0,51 \pm 0,01$ \\
Creatinina (mg/dl) & $0,70 \pm 0,05^{*}$ & $0,54 \pm 0,02$ \\
Proteínas totais (g/dl) & $4,90 \pm 0,07^{*}$ & $6,20 \pm 0,09$ \\
Triglicerídeos (mg/dl) & $117,77 \pm 21,27$ & $72,20 \pm 6,29$ \\
Colesterol total (mg/dl) & $65,68 \pm 4,68$ & $52,40 \pm 3,24$ \\
FA (U/l) & $41,34 \pm 6,59^{*}$ & $98,20 \pm 7,21$ \\
Glicose (mg/dl) & $134,74 \pm 4,49$ & $145,40 \pm 16,42$ \\
Potássio (mEq/l) & $6,32 \pm 0,45^{*}$ & $4,14 \pm 0,17$ \\
Sódio (mEq/l) & $143,00 \pm 0,54$ & $144,60 \pm 0,98$ \\
Uréia (mg/dl) & $40,26 \pm 5,63$ & $52,08 \pm 2,93$ \\
GGT (U/l) & $2,60 \pm 0,40^{*}$ & $5,00 \pm 0,00$ \\
\hline
\end{tabular}

$\mathrm{n}=$ número de animais por grupo; "Diferença estatisticamente significativa em relação ao grupo controle ( $\mathrm{p}<0,05$, ANOVA).
Tabela 4. Valores médios do leucograma ( \pm erro padrão da média) em ratos tratados com fitoterápico Cassaú Composto ${ }^{\circledR}$ na dose de $13 \mathrm{ml} / \mathrm{kg} / \mathrm{dia}$ (CC10) e um grupo controle tratado com o veículo do fitoterápico na dose de $13 \mathrm{ml} / \mathrm{kg} / \mathrm{dia}$ (CC).

\begin{tabular}{|c|c|c|}
\hline Variáveis & $\operatorname{CC} 10(n=5)$ & $\operatorname{CC}(n=5)$ \\
\hline $\begin{array}{l}\text { Contagem global } \\
\text { de Leucócitos }\left(\mathrm{mm}^{3}\right)^{\text {a }}\end{array}$ & $4540 \pm 373,3$ & $5040 \pm 342,4$ \\
\hline Neutrófilos bastonados (\%) & nihil & nihil \\
\hline Neutrófilos segmentados (\%) & $25 \pm 1,9^{*}$ & $37,2 \pm 3,2$ \\
\hline Eosinófilos (\%) & $1,8 \pm 0,4$ & $3,4 \pm 1,2$ \\
\hline Basófilos (\%) & nihil & nihil \\
\hline Linfócitos (\%) & $68,2 \pm 2,4$ & $58,6 \pm 3,7$ \\
\hline Monócitos (\%) & $1,2 \pm 0,4$ & $0,8 \pm 0,4$ \\
\hline \multicolumn{3}{|c|}{$\begin{array}{l}\mathrm{n}=\text { número de animais por grupo; }{ }^{\mathrm{a}} \mathrm{A} \text { contagem global de leucócitos } \\
\text { representa valores absolutos. A contagem de neutrófilos, eosinófilos, } \\
\text { basófilos, linfócitos e monócitos representa valores relativos (percen- } \\
\text { tuais) à contagem global de leucócitos; "Diferença estatisticamente } \\
\text { significativa em relação ao grupo controle }(\mathrm{p}<0,05) \text {. }\end{array}$} \\
\hline
\end{tabular}

Tabela 6. Níveis séricos da atividade do ácido úrico, ALT, AST, bilirrubina, creatinina, proteínas totais, triglicerídeos, colesterol total, FA, glicose, potássio, sódio, uréia e GGT em ratos tratados com o fitoterápico Cassaú Composto ${ }^{\circledR}$ na dose de $13 \mathrm{ml} / \mathrm{kg} / \mathrm{dia}$ (CC10) e um grupo controle tratado com o veículo do fitoterápico na dose de $13 \mathrm{ml} / \mathrm{kg} / \mathrm{dia}(\mathrm{CC})$. Dados expressos pela média absoluta (valores bioquímicos) \pm epm.

\begin{tabular}{lcc}
\hline Variáveis & CC10 $(\mathbf{n = 5})$ & CC $(\mathbf{n}=5)$ \\
\hline Ácido úrico (mg/dl) & $2,86 \pm 0,38^{*}$ & $1,84 \pm 0,14$ \\
ALT (U/l) & $37,81 \pm 7,90$ & $44,22 \pm 4,37$ \\
AST (U/l) & $57,51 \pm 3,18$ & $62,84 \pm 5,72$ \\
Bilirrubina total (mg/dl) & $0,49 \pm 0,03$ & $0,47 \pm 0,04$ \\
Creatinina (mg/dl) & $0,66 \pm 0,05^{*}$ & $0,84 \pm 0,01$ \\
Proteínas totais (g/dl) & $4,54 \pm 0,10^{*}$ & $6,26 \pm 0,12$ \\
Triglicerídeos (mg/dl) & $77,54 \pm 13,30$ & $51,80 \pm 10,07$ \\
Colesterol total (mg/dl) & $62,77 \pm 3,42$ & $54,60 \pm 2,93$ \\
FA (U/l) & $67,88 \pm 3,62^{*}$ & $104,80 \pm 15,40$ \\
Glicose (mg/dl) & $144,50 \pm 6,19$ & $138,60 \pm 7,08$ \\
Potássio (mEq/l) & $5,66 \pm 0,35$ & $5,04 \pm 0,11$ \\
Sódio (mEq/l) & $141,80 \pm 0,74$ & $139,60 \pm 0,25$ \\
Uréia (mg/dl) & $30,74 \pm 2,61$ & $34,80 \pm 1,17$ \\
GGT (U/l) & $2,60 \pm 0,40$ & $2,00 \pm 0,45$ \\
\hline
\end{tabular}

$\mathrm{n}=$ número de animais por grupo; "Diferença estatisticamente significativa em relação ao grupo controle ( $\mathrm{p}<0,05$, ANOVA). 
uma, cinco e dez vezes a dose recomendada para o tratamento da debilidade física, considera-se que no período de utilização de 30 dias o fitoterápico pode ser considerado atóxico. A administração do fitoterápico em dose única de $26 \mathrm{ml} / \mathrm{kg}$, utilizada no teste de toxicidade aguda, não desencadeou nenhuma morte, porém um sinal de toxicidade sistêmica foi observado (ataxia), de caráter transitório e reversível, onde este não interferiu no desenvolvimento ponderal dos animais, nos consumos de água e ração, bem como não provocou alterações macroscópicas nos órgãos internos dos animais experimentais.

Os sinais de toxicidade sistêmica são definidos a partir da redução na massa corporal dos animais experimentais. Além da redução do desenvolvimento ponderal, a toxicidade sistêmica se manifesta através da redução nos consumos de água e ração, alterações de comportamento, apatia e má condição da pelagem, como a presença de pêlos arrepiados [9,10]. Outros sinais de toxicidade podem se expressar pela alteração da massa relativa dos órgãos, alterações hematológicas e bioquímicas sangüíneas [5].

A avaliação bioquímica sangüínea de machos e fêmeas tratados com o fitoterápico Cassaú Composto ${ }^{\circledR 1}$ na maior dose $(13 \mathrm{ml} / \mathrm{kg} /$ dia $)$, demonstraram um perfil bioquímico sangüíneo dentro dos padrões normais para a espécie, a exceção de proteínas totais, que esteve abaixo dos valores normais e a uréia que esteve um pouco acima [2]. Diferenças significativas de determinadas enzimas e alguns metabólitos foram observados no grupo tratado, quando comparados ao grupo controle. Estas diferenças talvez possam ser explicadas pelo fato de que os limites de referência para determinadas enzimas e metabólitos sejam muito extensos [7]. O mesmo autor ainda cita que os limites de valores normais apropriados para a espécie, sexo e idade são particularmente importantes na análise individual de cada animal, onde a análise estatística não se limita apenas a comparação do grupo tratado e controle.

Neste trabalho, os níveis pouco elevados de uréia encontrados em ambos os sexos e grupos, não constituiu um indicador sensível de dano renal em roedores, já que a utilização de creatinina endógena constitui o mais específico e sensível teste para a avaliação renal, pois seu nível sangüíneo não é afetado pela dieta, idade e sexo [7]. Por outro lado, exames histopatológicos realizados não revelaram quaisquer alterações de parênquima e tecido renais, o que indicaria que este pequeno aumento estaria relacionado a fatores extra-renais (dieta).

$\mathrm{Na}$ avaliação hematológica, as alterações das séries leucocitárias não obtiveram correlação com o quadro clínico dos animais, uma vez que os animais permaneceram sadios durante todo o período experimental $[2,8]$.

\section{CONCLUSÕES}

Do conjunto de resultados, pode-se concluir que o fitoterápico Cassaú Composto ${ }^{\circledR}$ parece constituir um medicamento com uma boa margem de segurança, pois sua utilização até 10 vezes a dose terapêutica $(13 \mathrm{ml} / \mathrm{kg} / \mathrm{dia})$ recomendada pelo fabricante durante 30 dias, comprovou não produzir toxicidade sistêmica em ratos. A utilização de doses maiores $(26 \mathrm{ml} / \mathrm{kg} /$ dia) demonstrou ocasionar sinais de toxicidade, porém transitórios. Portanto o fitoterápico se enquadra na categoria de atóxico e/ou relativamente não prejudicial.

Agradecimentos. Este trabalho teve suporte financeiro da CAPES, PROPESQ/UFRGS e FAPERGS, além da Cibecol Industrial Farmacêutico LTDA.

\section{NOTAS INFORMATIVAS}

${ }^{1}$ Cibecol Industrial Farmacêutico Ltda., Porto Alegre, RS, Brasil.

\section{REFERÊNCIAS}

1 Agência Nacional de Vigilância Sanitária (ANVISA). 2004. Resolução nº 90, de 16 de março de 2004: Guia para realização de estudos de toxicidade pré-clínica de fitoterápicos. Brasília. ANVISA.

2 Anderson N.L. 1998. Criação e medicina básica dos animais de estimação de bolso. In: Birchard S.J. (Ed).Clínica de Pequenos Animais. São Paulo: Roca, pp.1523-1552.

3 Carvalho J.C.T. 2005. Formulário Médico-Farmacêutico de Fitoterapia. Belo Horizonte: Ciência Brasilis, pp.2-9.

4 Callegari-Jacques S.M. 2004. Bioestatística princípios e aplicações. São Paulo: Artmed Editora S.A., 255p.

5 González, F.H.D., Silva S.C. 2003. Introdução à Bioquímica Clínica Veterinária. Porto Alegre: UFRGS, pp.179-198.

6 Lapa A.J. 1999. Farmacologia e toxicologia de produtos naturais. In: Simões C.M.O. (Ed). Farmacognosia da planta ao medicamento. Florianópolis: Editora da Universidade Federal de Santa Catarina, pp.181-196. 
7 Loeb W.F. 1997. Clinical biochemistry of laboratory rodents and rabbits. In: Kaneko, J.J.(Ed). Clinical Biochemistry of Domestic Animals. 5th edn. San Diego: Academic Press, pp.845-855.

8 Lopes S.T.A. 1996. Patologia Clínica Veterinária. Santa Maria: CCR, 166p.

9 Mello F.B. 2001. Estudo dos efeitos de Lantana câmara (Verbenaceae) sobre a fertilidade e reprodução de ratos. 120f. Porto Alegre, RS. Dissertação (Mestrado em Ciências Veterinárias)-Programa de Pós-graduação em Ciências Veterinárias, Universidade Federal do Rio Grande do Sul.

10 Mello J.R.B., Langeloh A., Habermehl G., Krebs H.C., Batatinha M.J.M., Almeida C.C.R., Bastos F.C., Bassani M., Baruffaldi C., Alavares F., Francisco D. \& Kummer R. 1997. Avaliação do extrato aquoso dos frutos de Crotalaria retusa Leguminosae sobre a fertilidade de ratas. Arquivos da Faculdade de Veterinária UFRGS. 25:34-42.

11 Oga S. 1996. Fundamentos de toxicologia. São Paulo: Atheneu. 515p.

12 Osweiler G.D. 1998. Toxicologia Veterinária. Porto Alegre: Artes Médicas, 526p.

13 Sharapin N. 1996. Normatização da indústria farmacêutica. In: Anais do Seminário sobre industrialización y legislación de productos fitofarmacêuticos em iberoamérica (Guatemala, Guatemala). pp.42-44.

14 Triola M.F. 2003. Introdução à estatística. Rio de Janeiro: LTC Livros técnicos e científicos S.A., 410p.

15 Wojcikowski K., Johnson D.W. \& Gobé G. 2004. Medicinal herbal extracts - renal friend or foe? Part one: the toxicities of medicinal herbs. Nephrology. 9: 35-40. 